\title{
Progress in development of a new luminescence setup at the FinEstBeAMS beamline of the MAX IV laboratory
}

\author{
Vladimir Pankratov ${ }^{\mathrm{a}, \mathrm{b}, \mathrm{f}, *}$, Rainer Pärna ${ }^{\mathrm{a}, \mathrm{c}}$, Marco Kirm ${ }^{\mathrm{c}}$, Vitali Nagirnyi ${ }^{\mathrm{c}}$, Ergo Nõmmiste ${ }^{\mathrm{c}}$, \\ Sergei Omelkov ${ }^{c}$, Sebastian Vielhauer ${ }^{c}$, Kirill Chernenko ${ }^{\mathrm{a}}$, Liis Reisberg ${ }^{\mathrm{a}}$, Paavo Turunen ${ }^{\mathrm{b}}$, \\ Antti Kivimäki ${ }^{\mathrm{a}, \mathrm{b}}$, Edwin Kukk ${ }^{\mathrm{d}}$, Mika Valden ${ }^{\mathrm{e}}$, Marko Huttula ${ }^{\mathrm{b}}$ \\ ${ }^{\text {a } M A X ~ I V ~ L a b o r a t o r y, ~ L u n d ~ U n i v e r s i t y, ~ P . O . ~ B o x ~ 118, ~ 22100, ~ L u n d, ~ S w e d e n ~}$ \\ ${ }^{\mathrm{b}}$ Nano and Molecular Systems Research Unit, University of Oulu, P.O. Box 3000, FIN-90014, Oulu, Finland \\ ${ }^{\mathrm{c}}$ Institute of Physics, University of Tartu, W. Ostwald Str. 1, 50411, Tartu, Estonia \\ ${ }^{\mathrm{d}}$ Department of Physics and Astronomy, University of Turku, FIN-20014, Turku, Finland \\ ${ }^{\text {e }}$ Surface Science Group, Laboratory of Photonics, Tampere University of Technology, FIN-33101, Tampere, Finland \\ ${ }^{\mathrm{f}}$ Institute of Solid State Physics, University of Latvia, 8 Kengaraga, LV-1063, Riga, Latvia
}

\section{A R T I C L E I N F O}

\section{Keywords:}

Luminescence spectroscopy

Wide gap compounds

VUV

XUV photoexcitation

Synchrotron radiation

\begin{abstract}
A B S T R A C T
FinEstBeAMS is a new materials science beamline at the $1.5 \mathrm{GeV}$ storage ring of the MAX IV Laboratory in Lund, Sweden. It has been built based on grazing incidence monochromatization of synchrotron light, which allows to cover a remarkably wide excitation energy range from ultraviolet to soft x-rays (4.5-1450 eV). A new mobile luminescence spectroscopy end station has been commissioned with design benefitting from the advantages of a high flux elliptically polarizing undulator light source. We report on the design of the luminescence end station, its technical realization and performance achieved so far. Special attention is paid to the experimental challenges for luminescence spectroscopy under grazing incidence excitation conditions. The first luminescence results obtained demonstrate a reliable performance of the advanced setup at FinEstBeAMS.
\end{abstract}

\section{Introduction}

The tuneability of synchrotron radiation (SR) in a wide photon energy range and its inherent well-defined time structure makes it particularly well suited for time-resolved luminescence spectroscopy. A pioneering work on luminescence studies of ruby and $\mathrm{Cr}^{3+}$ doped $\mathrm{MgO}$ performed at the TANTALUS storage ring (Wisconsin, USA), pointing to a huge potential of synchrotron radiation based spectroscopy, was published in 1970 (Yen at al., 1970). This work was followed by luminescence studies at other synchrotron centres all over the world. The history, highlights and future of luminescence spectroscopy at synchrotron facilities have been discussed in several reviews through the years (see, e.g., Zimmerer, 2006; and Bel'skii et al., 2016). During the last three decades from 1982 until 2012, the SUPERLUMI setup at Strahl I of the DORIS III storage ring of HASYLAB at DESY, Hamburg, Germany (Gürtler et al., 1983) has been the flagship of luminescence research (Zimmerer 1991, 2007). Being a specially designed bendingmagnet beamline equipped with a normal incidence primary monochromator, Strahl I provided favourable experimental conditions for spectroscopy studies under VUV excitation to a remarkably large user community. Including the second Strahl I end station CLULU, dedicated to the fluorescence spectroscopy of atoms and clusters, the SUPERLUMI station produced as many as 1078 scientific publications in 30 years of their operation (Kotlov, 2018). Such a high scientific performance has demonstrated an obvious need for dedicated end stations which are specially designed for luminescence studies.

The luminescence and fluorescence spectroscopy has been an active research area also at the $500 \mathrm{MeV}$ storage ring MAX I of MAX Lab (Lund, Sweden) since its early days in the end of the 80's of the past century. The first beamline for time-resolved spectroscopy was put into operation in 1987 and used in fluorescence studies of biological molecular structures and their dynamics (Rigler et al., 1987). A few years later a research team from the Institute of Physics, Estonian Academy of Science, constructed a low-temperature photoluminescence setup at the bending magnet beamline 52 of the MAX I storage ring providing photons of 5-30 eV (Kink et al., 1991). A specially constructed cryostat was equipped with a device for crystal cleavage at ultra-high vacuum conditions. Utilizing a variety of luminescence methods, the research has been carried out on the relaxation processes of electronic excitations in simple and complicated oxides (Lushchik et al., 1994; Kisand

\footnotetext{
* Corresponding author. MAX IV Laboratory, Lund University, P.O. Box 118, 22100, Lund, Sweden.

E-mail address: vladimirs.pankratovs@maxiv.lu.se (V. Pankratov).
} 
et al., 1996; Nagirnyi et al., 1998), radiation defect formation in the conditions of the multiplication of electronic excitations (Lushchik et al., 1995), and the processes of secondary exciton formation in alkali halides (Lushchik et al., 1996). A linearly polarised VUV synchrotron radiation in the orbit plane was used to investigate polarization properties of intrinsic emissions and phosphorescence in tungstates (Feldbach et al., 2000; Nagirnyi et al., 2001).

The luminescence studies at MAX I continued until the commissioning of the 700-MeV MAX III storage ring in 2007 which, utilizing an elliptically polarizing undulator (EPU), provided radiation in the VUVXUV range (4.6-50 eV). A consortium consisting of Estonian (Tartu) and Finnish (Oulu, Turku and Tampere) universities built the FINEST branch-line at the I3 beamline of MAX III foreseen for gas phase (Urpelainen et al., 2010) and luminescence studies (Tuomela et al., 2016). The mobile low-temperature photoluminescence station was based on the VUV setup (Vielhauer, 2003), which was developed for studies of wide gap crystals under XUV excitation at the BW3 undulator beamline of HASYLAB at DESY (Kirm et al., 2003a). While used in Lund, two monochromators with different detectors covering a wide energy range of $1.2-8 \mathrm{eV}$ for luminescence analysis were applied at this setup. The examples of the luminescence research performed at this branch line comprise the studies of energy transfer processes in solid solutions of $\mathrm{Zn} / \mathrm{Mg}$ tungstates (Spassky et al., 2014), luminescence properties of intrinsic emissions and rare earth ions in fluorides (Ogorodnikov et al., 2015; Tuomela et al., 2016), luminescence of transition metal ions in borates (Romet et al., 2017), as well as the research on two-dimensional semiconductor systems (Pankratov et al., 2016), and ordinary and core shell nanoparticles (Kuzmanoski et al., 2015, 2016; Shirmane and Pankratov 2016; Shirmane et al., 2017).

The Finnish-Estonian Beamline for Atmospheric and Materials Science (FinEstBeAMS) is a new materials science beamline operating at the $1.5 \mathrm{GeV}$ storage ring of the MAX IV Laboratory inaugurated in 2016 outside of Lund in southern Sweden. The beamline was proposed and developed by the same consortium of Finnish and Estonian Universities which had previously constructed the branch-line at I3 of MAX III. The FinEstBeAMS was the first international beamline of the MAX IV Lab jointly funded by Estonian and Finnish investments. Due to its advanced magnetic lattice, the $1.5 \mathrm{GeV}$ ring provides excellent beam quality at low energies covering the spectrum from UV radiation to soft $\mathrm{X}$-rays. The beamline itself, due to its grazing incidence design, covers a wide photon energy range $4.5-1450 \mathrm{eV}$, and is therefore well suited to probe core and valence levels of matter in all states. One of the three FinEstBeAMS end stations is designed to perform luminescence studies of solids. It allows to perform reflection, transmission as well as luminescence measurements under VUV and XUV excitation in a wide temperature range. In the current article, we will discuss the design of luminescence setup, demonstrate its performance based on the results of the first experiments, and give an outlook of future developments.

\section{The FinEstBeAMS beamline}

A detailed design and expected performance of the FinEstBeAMS beamline was recently presented in (Pärna et al., 2017). In this section, we discuss only those beamline performance parameters which are relevant for optical and luminescence spectroscopy research. Currently, the beamline itself as well as the luminescence and gas phase end stations are under commissioning.

A long-period EPU is the photon source for the FinEstBeAMS beamline, providing photons from 4.3 to $1500 \mathrm{eV}$ according to the design values, which also sets the lowest energy limit for spectroscopic purposes. The EPU was built at the MAX IV Laboratory using a novel technique developed in-house and based on gluing permanent magnet pairs (Pärna et al., 2017). The magnet arrays of the EPU can be shifted with respect to each other in the longitudinal direction, which allows a full control over the radiation polarization. Table 1 summarizes the calculated total radiated power and low energy limits for different
Table 1

Summary of the calculated parameters of the elliptically polarizing undulator in different modes of operation. $\mathrm{h} \nu_{\min }$ is the minimal achievable photon energy. Total radiated power has been calculated with $500 \mathrm{~mA}$ ring current.

\begin{tabular}{lll}
\hline Polarization & $h v_{\min }(\mathrm{eV})$ & Total power $(\mathrm{kW})$ \\
\hline Horizontal & 4.1 & 2.4 \\
Vertical & 5.7 & 1.7 \\
Circular & 4.9 & 2.0 \\
Inclined $45^{\circ}$ & 9.6 & 0.9 \\
\hline
\end{tabular}

polarization modes. Due to the heat load of the first cooled optical elements, the total radiated power sets limits to the maximum ring current for small undulator gaps. Experiments proved that while operating the undulator with the closed gap of $14 \mathrm{~mm}$, its radiation spectrum at higher energies resembles that of a wiggler providing a continuous spectrum in the range of $1000-1450 \mathrm{eV}$. In the undulator mode, photon energies up to $1400 \mathrm{eV}$ can be reached.

The optical layout of the FinEstBeAMS beamline with the main optical elements is shown in Fig. 1. The first optical element is a sidecooled toroidal mirror M1 situated at a distance $12 \mathrm{~m}$ from the source. The amount of radiation accepted by M1 is controlled by baffles defining its vertical and horizontal acceptance. M1 collimates the beam in both vertical and horizontal directions. The grazing incidence monochromator of an SX700 type was manufactured by FMB (Feinwerk-und Messtechnik $\mathrm{GmbH}$, Berlin). It contains an internally cooled plane mirror M2 and two side-cooled plane gratings (PG1, 600 l/mm and PG2, $921 / \mathrm{mm}$ ), which cover high and low energy ranges from 15 to $1500 \mathrm{eV}$ and from 4.5 to $60 \mathrm{eV}$, respectively. A toroidal focusing mirror M3a focuses monochromatic radiation at the exit slit plane located at a distance of $6 \mathrm{~m}$. Thereafter, a single ellipsoidal mirror M4a is used to refocus the photon beam in both vertical and horizontal directions onto a sample located at the distance of $2.875 \mathrm{~m}$ from it. The simulated beam size on the sample is expected to be about $100 \times 100 \mu \mathrm{m}^{2}$ and it can be reduced further by closing baffles and the exit slit (see Table 2). For a more detailed discussion of the expected performance see (Pärna et al., 2017).

The calculated photon flux at a given resolving power $(R=5000)$ in the photon energy range of 4-1000 eV is shown in (Pärna et al., 2017). It is expected to be in the range from $10^{12} \mathrm{ph} / \mathrm{s}$ at $4.3 \mathrm{eV}$ to $10^{11} \mathrm{ph} / \mathrm{s}$ at $1000 \mathrm{eV}$ with the maximum value of $8 \cdot 10^{13} \mathrm{ph} / \mathrm{s}$ at $150 \mathrm{eV}$. The real flux measured with an AXUV-100G diode was of the order of $10^{11}$ for PG1 with $70-\mu \mathrm{m}$ slits and $10^{12} \mathrm{ph} / \mathrm{s}$ for PG2 with $30-\mu \mathrm{m}$ slits (Fig. 2 (a)). It is in reasonable agreement with the calculated values. It is important to point out that no filters reducing higher orders of radiation were applied in these measurements. Therefore, the recorded flux values contain currently undetermined contribution of the higher undulator harmonics passing through the primary monochromator in a higher diffraction order of the grating. The estimation of such contribution at different energies is not a trivial task because of a grazing incidence geometry used in a SX-700 type monochromator. Here, the angle of incidence at the plane mirror M2 can is varied in the range of $90-70^{\circ}$, the grazing incidence angle is $11.2^{\circ}$ for PG1, which provides a more efficient higher order suppression in respect of PG2 with a grazing incidence angle of $4.4^{\circ}$ at $25 \mathrm{eV}$ (Pärna et al., 2017). Thus, the grazing incidence optical scheme in combination with an undulator source introduces a serious experimental challenge related to the suppression of higher order radiation. The same higher order suppression problem has occurred at the studies of the multiplication of electronic excitations in alkali halides (Beaumont et al., 1976) and in MgO (Vasilev et al., 1985), which have also been performed using grazing incidence monochromators. In the case of luminescence studies carried out at normal incidence instruments (incl. former setups in Lund and in Hamburg), a low reflectivity of metal coatings of mirrors and gratings set the higher energy limits to 20 and $40 \mathrm{eV}$ for the $\mathrm{Al}$ and Pt coated gratings, respectively (see Fig. 2 (a)). At such setups, the second order radiation is 


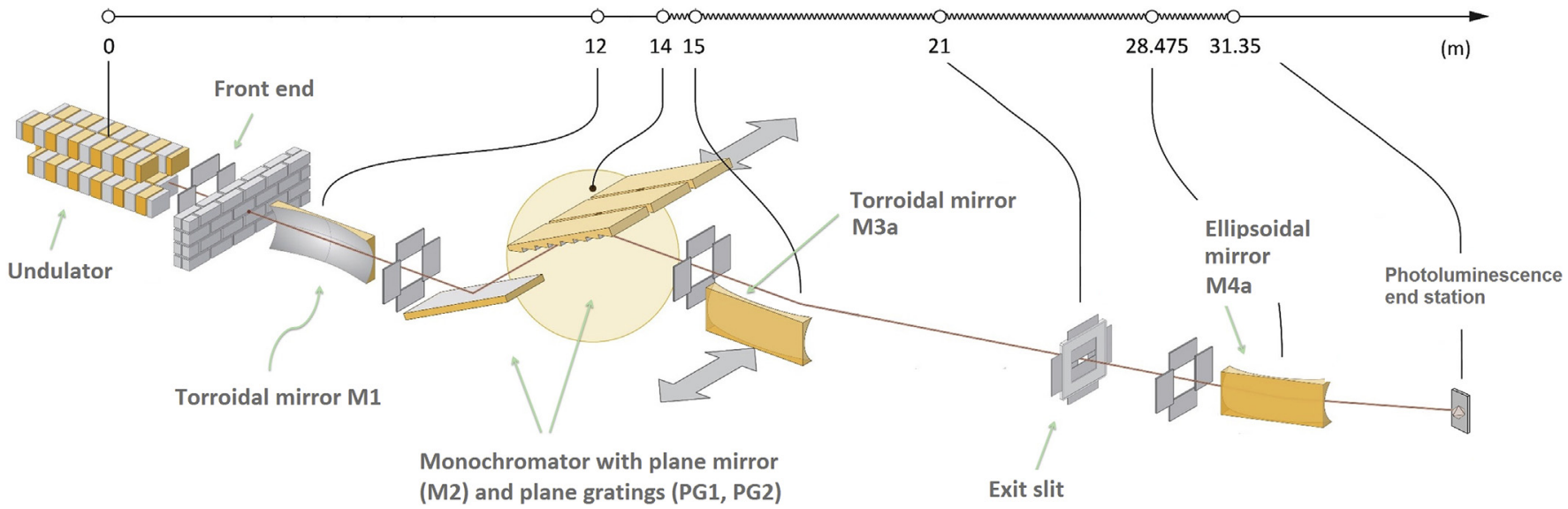

Fig. 1. The optical layout of the branch of the FinEstBeAMS beamline where photoluminescence setup will be finally installed. Relevant passive and active optical elements are shown together with a length scale.

removed by inserting optical windows with transmission edges in the VUV range (usually $\mathrm{SiO}_{2}, \mathrm{MgF}_{2}$ and $\mathrm{LiF}$ ) into synchrotron radiation beam. In order to suppress high orders of excitation at FinEstBeAMS beamline, operating in an exceptionally wide energy region, a set of filters has been chosen to overcome this problem. Fused silica and LiF filters transparent up to 8 and $10.8 \mathrm{eV}$, respectively, are chosen for the low energy region. At higher energies, thin films of $\mathrm{Sn}(4 \mathrm{~d} \mathrm{BE} 23.9 \mathrm{eV})$, $\mathrm{Mg}$ (2p BE 49.6 eV), Al (2p BE $72.5 \mathrm{eV}$ ), Si (2p BE $99.2 \mathrm{eV}$ ), B (1s BE $188 \mathrm{eV}$ ) and $\mathrm{C}(1 \mathrm{~s} \mathrm{BE} 284.2 \mathrm{eV}$ ) can be used as filters with the transparency region edge defined by the binding energy (BE) of the corresponding electronic state indicated in brackets. These filters cover almost the whole operation range of the first undulator harmonic. A specific characterization of each filter will be performed during commissioning of the beamline and end stations.

In Fig. 2 (a), photon fluxes for the $\mathrm{Al}$ and Pt coated gratings (1200 l/ $\mathrm{mm}$ ) of the SUPERLUMI station (Zimmerer, 2007) are shown. Despite a large $50 \mathrm{mrad}$ acceptance of bending magnet radiation the photon flux available is noticeably lower than that recorded at FinEstBeAMS. This shows the advantage of beamlines using an undulator source. The use of an undulator source has been also proposed for upgrade of SUPERLUMI station by Prof. Zimmerer, but it was never realized at DESY. It is obvious that such powerful high energy radiation sources will enable combination of different research methods, which require either high photon flux or high spectral resolution. For example, an even more complex conceptual design of the setup for luminescence and EPR spectroscopy has been proposed, where short wavelength radiation from insertion devices at storage rings or free electron lasers is to be used (Makhov and Kolobanov 2001). The luminescence spectroscopy of solids does not need such high resolution in all cases, so an even more intense photon flux can be used instead to study in materials processes with low quantum efficiency or to create radiation defects. The defect formation processes in radiation sensitive alkali halides have been discussed in a recent review (Lushchik, A. and Lushchik, Ch., 2018) and such studies can be accomplished using advanced light sources.

In order to provide continuously changing intensity of incident radiation required in recording excitation, reflection and transmission spectra, the undulator gap has to be changed together with the incident photon energy to ensuring the highest flux. This task is realized in the experiment control program by using a software algorithm, which optimizes the insertion device gap based on the desired photon energy, according to reference values determined earlier experimentally during the commissioning process. Fig. 2 (b) shows that the undulator peaks near the low energy limit (recorded without any filter) become narrower and their intensity significantly lowers as the EPU gap is decreased. This reduction in intensity and bandwidth of undulator peaks is caused by the wider divergence of the undulator radiation for smaller energies and the constant acceptance of the monochromator. Another significant reason in a remarkable transformation of the peak shape detected in our experiment is due the presence of undulator higher order harmonics which possess sufficiently high intensity, and are situated in the region of enhanced grating efficiency.

The main parameters of the undulator, optical elements, monochromator and the photon beam of FinEstBeAMS in general are summarized in Table 2.

\section{Setup for luminescence studies}

The FinEstBeAMS beamline is designed for a multidisciplinary research and contains several end stations installed on the two branch lines. One of these is a compact setup for low temperature luminescence studies, a schematic view of which is presented in Fig. 3. The designed luminescence setup is a mobile end station easily useable at other MAX IV beamlines or operated using external excitation sources. When the luminescence setup is detached, other user-developed experimental stations and instruments can be attached to the FinEstBeAMS beamline instead. A special care is taken in the design of a rail system, which allows smooth mounting and alignment of the instruments.

The main UHV chamber of the luminescence setup has a spherical shape $26 \mathrm{~cm}$ in diameter. It is evacuated to working vacuum level $10^{-9}$ mbar at room temperature by a turbo pump HiPace 300 (Pfeiffer

Table 2

Summary of design parameters of the FinnEstBeaMS beamline.

\begin{tabular}{|c|c|}
\hline Radiation source (EPU) & Elliptically polarizing undulator, $95.2 \mathrm{~mm}$ period, 26 periods, $14 \mathrm{~mm}$ minimum gap, $2.475 \mathrm{~m}$ magnetic length \\
\hline Energy (wavelength) range & $4.4-1000 \mathrm{eV}(288-1.24 \mathrm{~nm})$ \\
\hline Monochromator (cPGM) & Plane grating monochromator using collimated light, two plane gratings (92 and $600 \mathrm{~mm} / \mathrm{l}$ ), internally cooled planar premirror \\
\hline Maximal resolving power & $\mathrm{R}=\mathrm{E} / \Delta \mathrm{E} \approx 1 \times 10^{4}$ \\
\hline (Re)Focusing optics & Ellipsoidal mirror \\
\hline Beam size at sample & $\begin{array}{l}100 \times 100 \mu \mathrm{m}^{2} \text { (horizontal } \mathrm{x} \text { vertical), } 30 \times 20 \mu \mathrm{m}^{2} \text { when limiting exit slit and baffles. The beam size can be increased by moving the luminescence } \\
\text { end station out of focus }\end{array}$ \\
\hline Flux at sample & $10^{11-8} 8 \times 10^{13}-\mathrm{ph} / \mathrm{s}$ \\
\hline
\end{tabular}



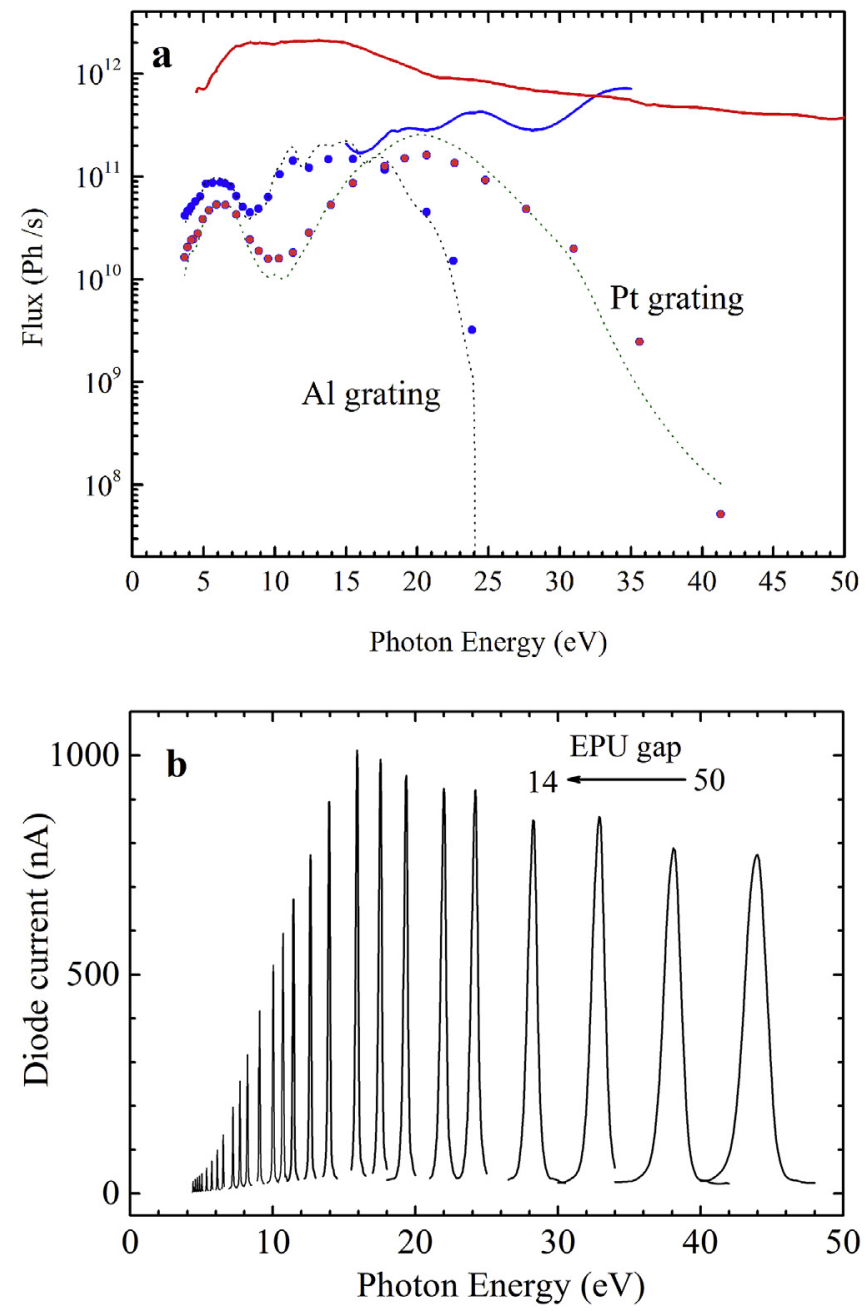

Fig. 2. a) A recorded photon flux for 92 (PG2, red line) and 6001/mm (PG1, blue line) grating. For comparison a typical photon flux at the SUPERLUMI station for the $\mathrm{Al}$ and Pt coated gratings are presented. (b) A set of undulator radiation spectra as a function of its gap width. (For interpretation of the references to colour in this figure legend, the reader is referred to the Web version of this article.)

Vacuum) and ion pump (Gamma Vacuum), which are mounted on the bottom of the chamber. Since the setup is located behind the end station for gas phase studies, there is an additional small turbo pump HiPace 80 (Pfeiffer Vacuum) used to prevent potential residual gas contamination from the gas phase experiment. A residual gas analyser will be installed to monitor vacuum quality in the main chamber.

The UHV chamber is equipped with a helium cryostat with a coldfinger type sample holder. The closed-cycle helium cryostat consists of a ARS DE-204PB cryohead and the ARS-4HW compressor (Advanced Research System). A LakeShore 335 temperature controller allows to regulate sample temperature in the region of $6-400 \mathrm{~K}$. The cryostat, mounted on a rotational stage, is installed on a XYZ manipulator (Thermionics) facilitating the alignment of the sample with respect to the incident beam. The size $\left(12 \times 2 \mathrm{~cm}^{2}\right)$ of the two-sided sample holder is large enough to accommodate dozens samples on each side. Mounting numerous samples in one load helps to minimize time for sample exchange and system bake-out.

The setup is designed for luminescence analysis with two secondary monochromators (Fig. 3). Luminescence detection in UV-visible-NIR spectral range (200-1500 nm) can be performed by an Andor Shamrock (SR-303i) $0.3 \mathrm{~m}$ spectrometer equipped with a high resolution grating $(1200 \mathrm{l} / \mathrm{mm}, 300 \mathrm{~nm}$ blaze) and two lower resolution gratings (300 l/ $\mathrm{mm}$ ) optimized for different spectral regions (300 and $500 \mathrm{~nm}$ blaze, respectively). The Andor Shamrock spectrometer collects luminescence from samples with the aid of a fibre optic cable. The cable end can be positioned in the UHV experimental chamber using a 3-axis manipulator to achieve the highest signal intensity. Such solution has been earlier successfully implemented at luminescence research performed at the MAX III storage ring (Tuomela et al., 2016). It was shown that materials with different quantum efficiencies of luminescence can be successfully studied using such technical approach.

The Andor Shamrock spectrometer has two exit ports equipped with a CCD detector (Newton DU9 70P-BVF model from Andor) and a photomultiplier, respectively. Currently there is a set of easily replaceable photomultipliers (H8259, H8259-01, and H8259-02 Hamamatsu photon counting heads) covering the spectral range from 185 to $900 \mathrm{~nm}$ and a thermoelectrically cooled infrared NIR PMT Unit H10330C-45 (Hamamatsu) for the spectral range of 950-1400 nm. These detectors have rather slow time response (tens of ns) and therefore are wellsuited for steady-state luminescence measurements, but not for timeresolved measurements in the timing conditions provided by the $1.5 \mathrm{GeV}$ storage ring of MAX IV. At present, it operates in multi-bunch mode (32 bunches of 130 ps duration with $10 \mathrm{~ns}$ interval). A singlebunch mode with a 320 ns time interval of the bunches of 40 ps duration is also planned for the nearest future (Olsson et al., 2018). In view of this, the development of a fast sub-ns luminescence registration system is in progress at the FinEstBeAMS beamline.

The design of the luminescence setup foresees also the possibility of developing a VUV branch based on a monochromator with the following design parameters: $0.4 \mathrm{~m}$ focal length, $0.6 \mathrm{~nm}$ resolution, $1650 \mathrm{l} / \mathrm{mm}$ grating, and wavelength range $50-300 \mathrm{~nm}$.

Transmission and reflection spectra can also be recorded at the FinEstBeAMS beamline. Two AXUV-100G diodes mounted in UHV serve as detectors of either transmitted or reflected radiation from the samples in suitable geometry. The angle of incidence in the reflection geometry is $27.5^{\circ}$. There is also an additional viewport available at the angle of $45^{\circ}$, which can be utilized for photo-stimulated luminescence experiments (see, e.g., Lushchik et al., 1996) or for pump-probe spectroscopy by means of external light sources. The incident flux used for normalization of the spectra is recorded with another AXUV-100G diode alongside with the photocurrent from the M4a mirror as a function of scanned photon energy. It is corrected with the diode's quantum efficiency and divided by the M4a photocurrent signal. While scanning the primary monochromator in the measurements of reflectivity and luminescence excitation spectra, the signal from the sample is also corrected for the spectral distribution of the incident photon flux and for the photocurrent from the M4a mirror, reflecting the changes in the flux during the measurement time. This method has been developed and tested earlier for the luminescence measurements under the XUV excitation performed at the BW3 beamline of HASYLAB (Kirm et al., 2003a). Our preliminary tests at the FinEstBeAMS beamline indicated that sodium salicylate, which is typically used for recording photon flux under VUV excitation, is not stable under high photon flux provided by FinEstBeAMS and degrades noticeably during the measurement time. Therefore, the above-described more reliable spectral correction methods are used at the beamline. Nevertheless, the normalization of the excitation spectra in the whole energy range extending from 4.5 to $1450 \mathrm{eV}$ is a serious challenge and the corresponding methods and reliable procedures have yet to be worked out during the commissioning process.

\section{The first experimental results and future developments at FinEstBeAMS beamline}

The first photoluminescence experiments were carried out in the early commissioning phase of the FinEstBeaMS beamline. Although the design value of the low energy limit for the FinEstBeaMS undulator radiation is $4.3 \mathrm{eV}$ to meet the requirements of luminescence research, 


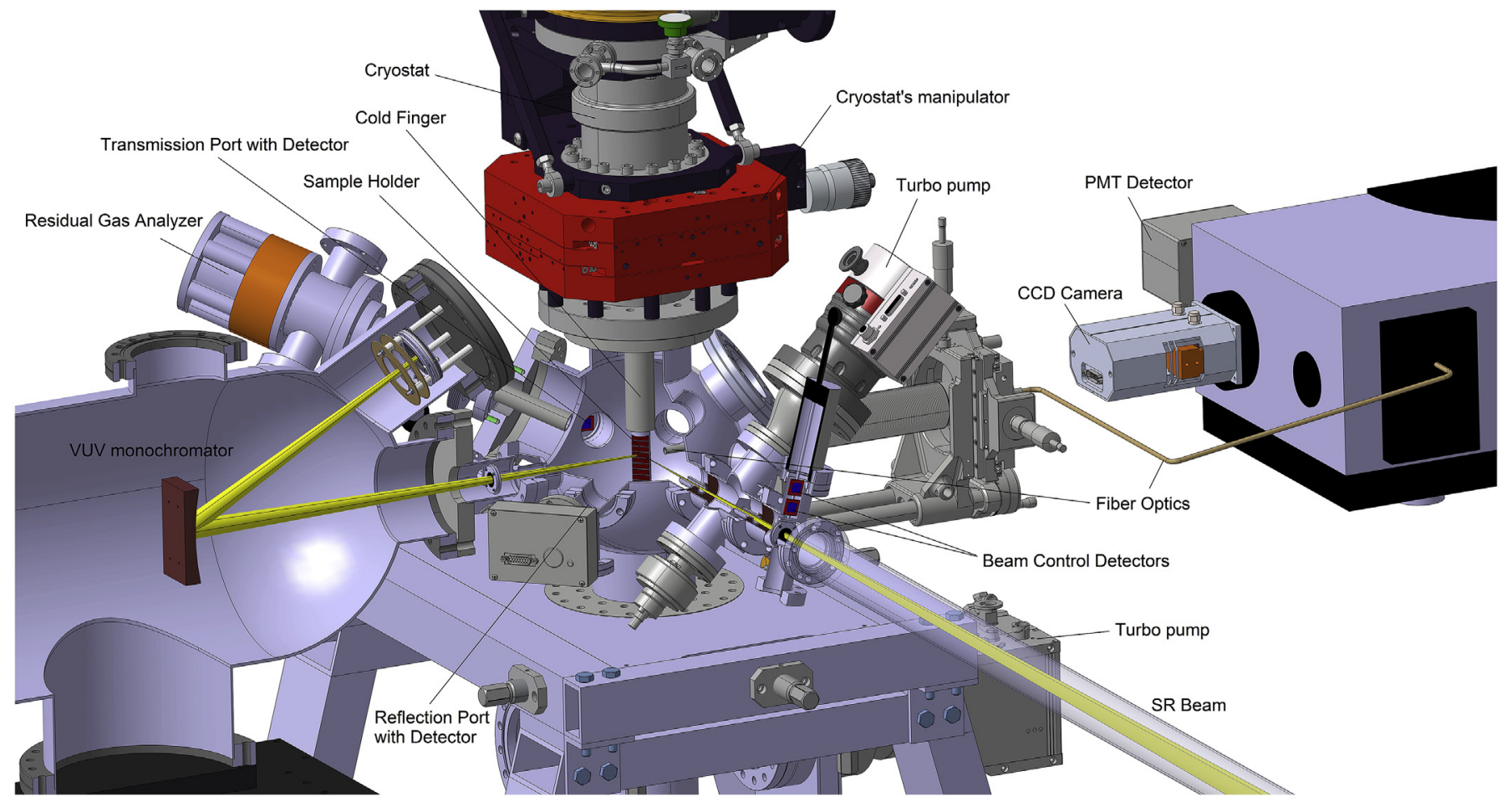

Fig. 3. A scheme of the luminescence setup at the FinEstBeAMS beamline.

only the PG1 grating setting the lowest photon energy limit to $15 \mathrm{eV}$ was available in first experiments. This condition determined also the selection of samples which were studied in the commissioning process. In the photon energy range of $15-35 \mathrm{eV}$, the formation of cation excitations (Rubloff, 1972), the processes of multiplication of electronic excitations (Lushchik et al., 1996; Lushchik, A. and Lushchik, Ch., 2018), and the relaxation of hot charge carriers in alkali halides and simple oxides (Kirm et al., 1999a) have been earlier studied, providing an accurate comparison material for the results obtained at a new experimental setup. At this early stage of the experiment development, no spectral corrections to the measured emission spectra were introduced.

Fig. 4 depicts the emission (a) and excitation spectrum (b) of the $\mathrm{Y}_{3} \mathrm{Al}_{5} \mathrm{O}_{12}: \mathrm{Ce}^{3+}$ crystal at room temperature. The YAG:Ce single crystal was grown by CRYTUR Ltd. As shown in Fig. 4(a), the emission spectrum displays the typical luminescence in the yellow spectral region of this compound with a high quantum yield, which nowadays serves as a LED phosphor in "cool" white light LEDs (Krames et al., 2007). Due to the parity and dipole allowed $5 \mathrm{~d}-4 \mathrm{f}$ transitions of the $\mathrm{Ce}^{3+}$ ions, this material serves also as a fast scintillator with a decay time of few tens of ns (Kirm et al., 2000). The excitation spectrum in Fig. 4 (b) demonstrates that the intensity of $530 \mathrm{~nm}$ emission increases more than twice in the energy range from 15 to $35 \mathrm{eV}$. This is to a certain extent due to a small decrease of the host absorption coefficient (more $\mathrm{Ce}^{3+}$ centres in the crystal volume become excited), but the main reason is the multiplication of electronic excitation since the energy gap value of this material is $8 \mathrm{eV}$ (Kirm et al., 2000; Pankratov et al., 2008). The origin of the spectral features in the region of $28-34 \mathrm{eV}$ needs further investigation, as the transitions from the $2 \mathrm{p}^{6}(26-29 \mathrm{eV})$ and $2 \mathrm{~s}^{2}$ shell $30-37 \mathrm{eV}$ ) of the oxygen ion are expected in this region as discussed for $\mathrm{Al}_{2} \mathrm{O}_{3}$ in (Kirm et al., 1999b). A general agreement between the two excitation spectra recorded for the $530-\mathrm{nm}$ emission in different experiments is good. The main difference that is observed above $27 \mathrm{eV}$ may be due to the normalization problems in previous measurements at the bending magnet beamlines, at which the correction of excitation spectra is less reliable in a high-energy region, where the incident photon flux becomes weaker (see Fig. 2 (a)).

Rare-earth (RE) ion doped materials are indispensable in many modern luminescence applications. The $4 \mathrm{f}-4 \mathrm{f}$ and $4 \mathrm{f}-5 \mathrm{~d}$ transitions of RE ions cover a wide energy range from IR to VUV, the study of which requires experimental facilities at storage rings. For instance, the quantum cutting processes providing more than one low energy photon per one absorbed VUV photon were studied in the $\mathrm{LiGdF}_{4}$ :Eu compound (Wegh et al., 1999), which is of special interest for mercury-free lightening based on short wavelength Xe discharge. Alongside with the identification of the application potential, the fundamental properties of the $4 \mathrm{f}-4 \mathrm{f}$ and $5 \mathrm{~d}-4 \mathrm{f}$ luminescence of RE ions were studied systematically at HIGITI (see e.g., Pieterson et al., 2002a and 2002b) and SUPERLUMI (Becker et al., 1998; Chen et al., 2003; Kirm et al., 2004). This justifies the choice of a $\mathrm{LiYF}_{4}$ :Er crystal as a model system with well described luminescence properties (see Khaidukov et al., 2002). The crystal used in this work has the same origin. The emission spectrum of the $\mathrm{LiYF}_{4}$ : $\mathrm{Er}(1 \%)$ single crystal under $44.2 \mathrm{eV}$ photon excitation at $300 \mathrm{~K}$ is shown in Fig. 5 (a), and the room temperature excitation spectrum for the ${ }^{4} \mathrm{~S}_{3 / 2}-{ }^{4} \mathrm{I}_{15 / 2}$ transition recorded at $550 \mathrm{~nm}$ is shown in Fig. 5 (b). There is a good agreement with the results obtained in (Khaidukov et al., 2002) for the band-to-band excitation of the host, although the spectra have been recorded at different temperatures. Fig. 5 (a) demonstrates a high quality of emission spectra recorded in a wide wavelength range extending from 200 to $1100 \mathrm{~nm}$ using the photoluminescence setup at the FinEstBeAMS beamline.

The coexistence of self-trapped and free excitons is an intrinsic property of electronic excitations in a number of wide gap solids (Song and Williams, 1996). Taking the advantage of time-resolved photoluminescence spectroscopy under SR excitation, the peculiarities of formation processes of excitons, their self-trapping, prompt and delayed formation of secondary excitons have been studied in rare gas solids (Kirm et al., 2003b) and in alkali halides, in which the relaxation dynamics and morphology of self-trapped excitons has been clarified (Kan'no 1996). The research of these fundamental properties in wide gap materials of various morphologies (incl. low-dimensional and nanomaterials) will fill a significant amount of beamtime available at the photoluminescence end station at FinEstBeAMS.

Among the intrinsic emissions there is a special kind of emission resulting from the recombination of an electron in the valence with a 

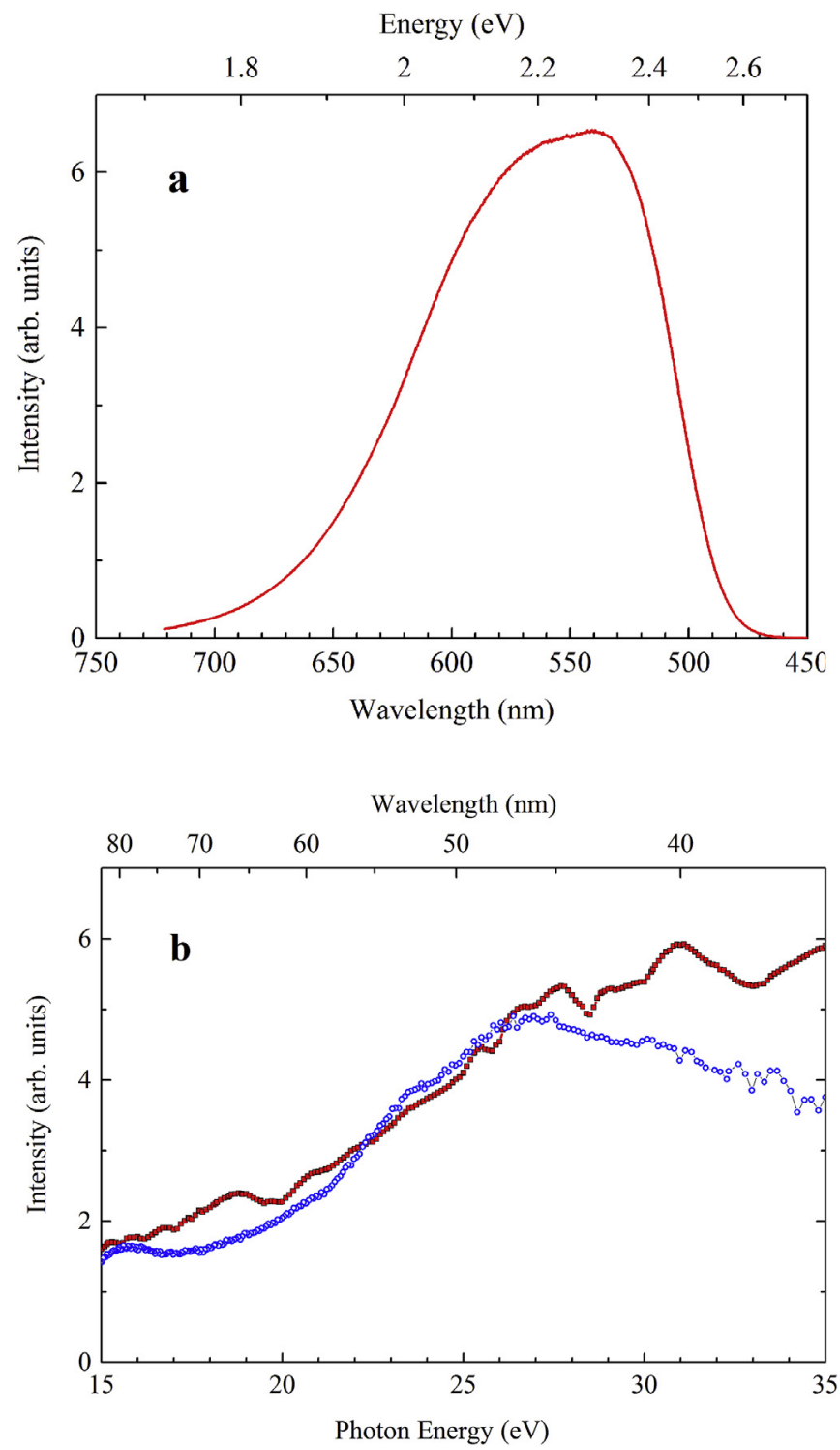

Fig. 4. (a) The emission spectrum of YAG:Ce (Crytur, Czech Republic) crystal at $300 \mathrm{~K}$ excited by $35 \mathrm{eV}$ photons at the FinEstBeAMS beamline. (b) The excitation spectra of the same YAG:Ce sample for $530 \mathrm{~nm} \mathrm{Ce}^{3+} 5 \mathrm{~d}-4 \mathrm{f}$ emission (red $\square$ ) measured at $300 \mathrm{~K}$. For comparison an analogous excitation spectrum of the $5 \mathrm{~d}-$ $4 \mathrm{f}$ emission of $\mathrm{Ce}^{3+}$ ion (blue o) from (Kirm et al., 2000) recorded at SUPERLUMI is presented. (For interpretation of the references to colour in this figure legend, the reader is referred to the Web version of this article.)

hole in the outermost core band, which takes place only in certain materials in which the forbidden energy gap is larger than the separation of the valence and the outermost core band (Makhov, 2014). This luminescence has several names like cross-luminescence (CL), corevalence luminescence or Auger-free luminescence (Kubota et al., 1988). Its nature has been proven for $\mathrm{BaF}_{2}$ by synchrotron radiation experiments which have shown that the CL is excited after the formation of the hole in the outermost core band (Aleksandrov et al., 1984). A typical decay time of $\mathrm{CL}$ is in the nanosecond time range and in $\mathrm{BaF}_{2}$ its value equals $0.92 \mathrm{~ns}$ (Terekhin et al., 1995). Fig. 6 (a) demonstrates the emission spectrum of $\mathrm{BaF}_{2}$ single crystal under the excitation by the $35 \mathrm{eV}$ photons, recorded in a time-integrated mode at room temperature. The dominant emission band peaked at $330 \mathrm{~nm}$ is due to the radiative recombination of self-trapped excitons. On the long-wavelength side, it is followed by a defect-related emission centred near $400 \mathrm{~nm}$. The UV spectrum part displays the CL with a maximum at $230 \mathrm{~nm}$ as
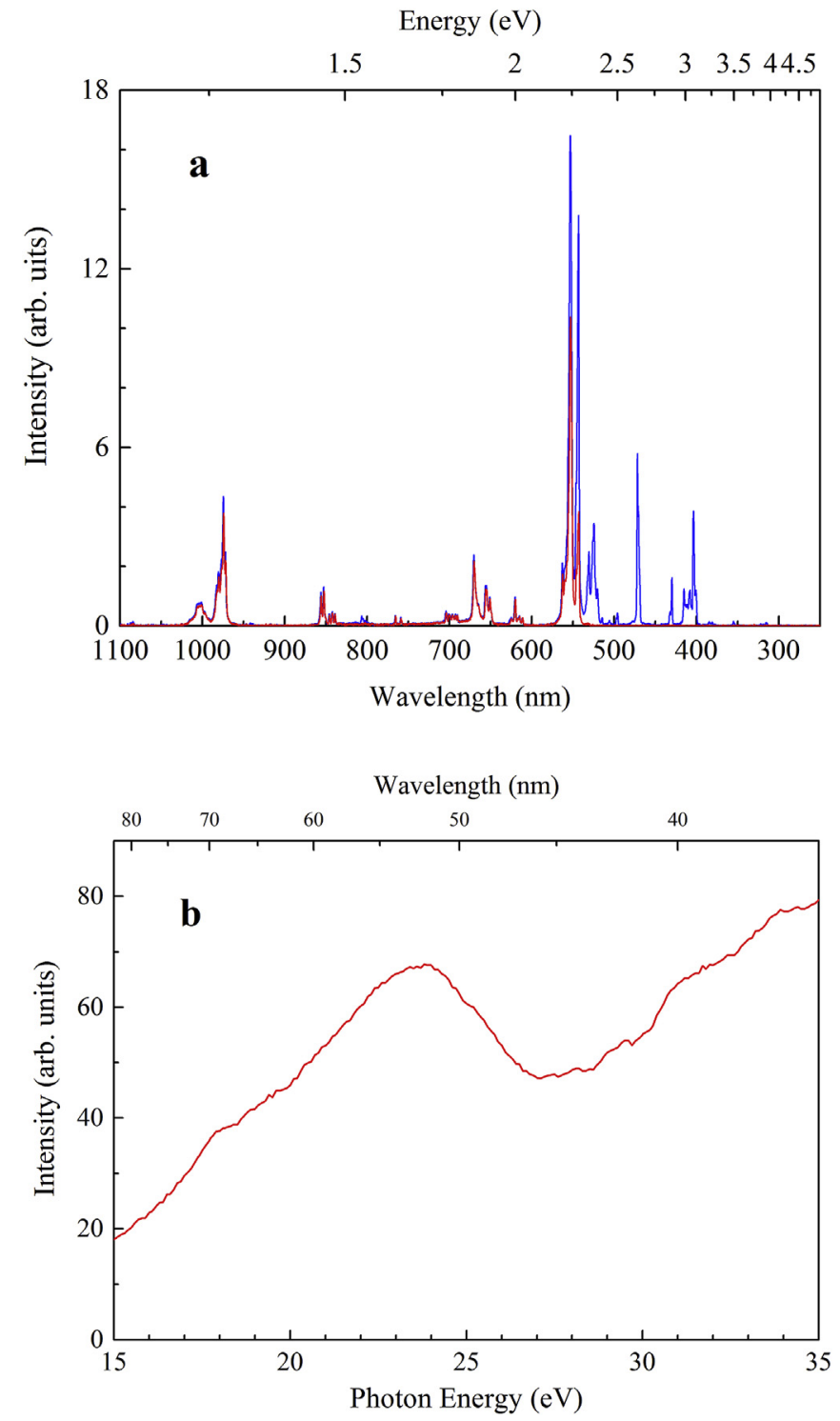

Fig. 5. (a) The emission spectrum of $\mathrm{LiYF}_{4}: \mathrm{Er}$ crystal excited by $44.2 \mathrm{eV}$ photons at $300 \mathrm{~K}$ recorded with the $(300 \mathrm{l} / \mathrm{mm}$ blazed at $300 \mathrm{~nm}$ ) grating without (blue line) and with the FLG550 filter (red line) at the FinEstBeAMS beamline. (b) The excitation spectra of $\mathrm{LiYF}_{4}$ :Er crystal for $550 \mathrm{~nm} \mathrm{Er}^{3+} 4 \mathrm{f}^{4} \mathrm{~S}_{3 / 2}-{ }^{4} \mathrm{I}_{15 / 2}$ emission (red line) at $300 \mathrm{~K}$ at the FinEstBeAMS beamline. (For interpretation of the references to colour in this figure legend, the reader is referred to the Web version of this article.)

expected (Makhov, 2014). Fig. 6 (b) shows the excitation spectra for the CL emission at $230 \mathrm{~nm}$ recorded at the setups in Lund and earlier in Hamburg (red and blue symbols, respectively). The overall agreement of both spectra is good and the onset of CL excitation occurs right below $18 \mathrm{eV}$, which corresponds to the ionization threshold for the $5 p \mathrm{Ba}^{2+}$ core band. The maxima in the reflection spectrum (black line) of $\mathrm{a} \mathrm{BaF}_{2}$ are correlated with the features in the excitation spectra of CL. Such comparison provides a solid argument in favour of high beamline quality and its suitability for the photoluminescence research at the FinEstBeAMS.

Finally, a few remarks about the upcoming time-resolved measurements at the FinEstBeAMS. As mentioned above, a new sub-nanosecond luminescence registration system is being developed at the FinEstBeAMS beamline in a configuration especially suited for the reduced bunch operation mode with 40 ps pulse width and 320 ns interval between bunches. Taking into account the high brilliance of the storage ring this new beamline will be especially suitable for the study 

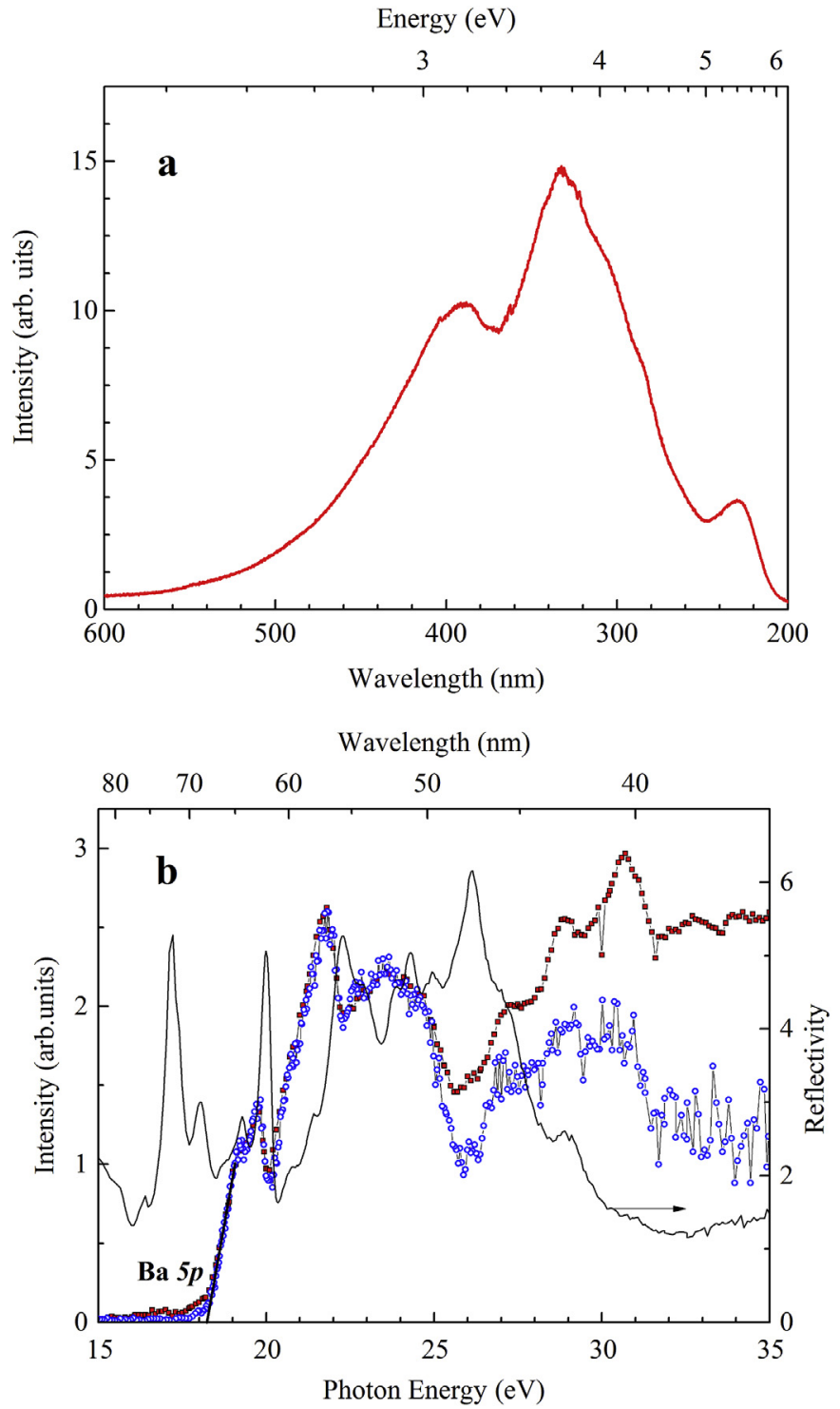

Fig. 6. (a) The emission spectrum of $\mathrm{BaF}_{2}$ crystal excited by $35 \mathrm{eV}$ photons at $300 \mathrm{~K}$. (b) The excitation spectrum of CL recorded (red $\square$ ) at $230 \mathrm{~nm}$ of a $\mathrm{BaF}_{2}$ crystal at $300 \mathrm{~K}$ (PG2). For comparison a similar excitation spectrum of the CL (blue o) and the reflection spectrum recorded (black line) recorded at SUPERLUMI at $8 \mathrm{~K}$. (For interpretation of the references to colour in this figure legend, the reader is referred to the Web version of this article.)

of fast and weak emissions, which are of great importance for upcoming timing applications. Medical applications such as time-of-flight positron emission tomography (TOF-PET) and high luminosity experiments in high energy physics demand novel scintillators, whose characterization requires sub-nanosecond time resolution and high brilliance due to a low intensity of the ultra-fast emissions. The FinEstBeAMS provides a high photon flux in a wide photon energy range, well suited for the studies of the creation and relaxation processes of electronic excitations under excitation in the characteristic energy regions (i.e., valence excitations at the edge of intrinsic absorption, inner shell excitation by high energy photons, etc). Further commissioning experiments will determine the weakest phenomena which can be studied. Recently, there has been a strong interest towards an intra-band luminescence (Lecoq et al., 2014; Omelkov et al., 2016, 2018), which is due to the transitions between electronic states within the conduction or valence band with a quantum yield of $10^{-4}$. So far, the investigations of such weak emissions have been mostly performed using powerful electron beams (e.g., Omelkov et al., 2018; Grigorjeva et al., 2001). We suppose that, due to the sub-nanosecond time excitation pulses, synchrotrons with high photon flux and free electron lasers can help to reveal the peculiarities of such ultrafast luminescence phenomena.

\section{Conclusions}

A new luminescence end station has been developed and successfully installed at the FinEstBeAMS beamline at MAX IV Laboratory. It is designed for the studies of the optical and luminescence properties of various types of inorganic solids ranging from classical single crystals to modern nanomaterials and composite compounds. The beamline is currently under commissioning. Its technical configuration allows to study the following characteristics of wide gap materials at temperatures 7-400 K under synchrotron radiation generated by an elliptically polarizing undulator: i) transmission, reflection and excitation spectra in a wide photon energy range $4.5-1450 \mathrm{eV}$ utilizing a grazing incidence plane grating monochromator for high resolution studies; ii) polarization and dichroism effects using the ability of the undulator to produce radiation with fully controllable polarization (horizontal, vertical, circular); iii) emission spectra from UV to NIR spectral range (200-1500 nm); iv) decay kinetics of emission in both standard multi bunch (10 ns interval) and single bunch (320 ns interval) modes of the storage ring.

The above-presented results of the first preliminarily commissioning experiments at the FinEstBeAMS beamline, although performed in a relatively small range of excitation energies, demonstrated the high potential of the photoluminescence setup in time-resolved spectroscopic studies of wide gap solids and its ability to attract considerable attention of the scintillator and phosphor research community.

\section{Acknowledgements}

The main funding for the FinEstBeAMS beamline has been obtained from the European Union through the European Regional Development Fund (project "Estonian beamline to MAX-IV synchrotron", granted to the University of Tartu) and from the Academy of Finland through the Finnish Research Infrastructure funding projects (FIRI2010, FIRI2013, FIRI2014). The authors also acknowledge the funding contributions of the University of Oulu, University of Turku, Tampere University of Technology, the Estonian Research Council (IUT 2-25, IUT 2-26, PRG111), as well as the Estonian Centre of Excellence in Research "Advanced materials and high-technology devices for sustainable energetics, sensorics and nanoelectronics" TK141 (2014-2020.4.01.150011). The strategic funding of Finnish FIMAX consortium coordinating university, University of Oulu, for beamline personnel is also acknowledged. The authors thank the MAX IV Laboratory for financial and infrastructural support as well as for assistance during the construction of the FinEstBeaMS beamline.

\section{References}

Aleksandrov, Yu.M., Makhov, V.N., Rodnyj, P.A., Syrejshchikova, T.I., Yakimenko, M.N., 1984. Intrinsic luminescence of $\mathrm{BaF}_{2}$ at pulsed synchrotron radiation excitation. Phys. Sol. State 26, 1734.

Beaumont, J.H., Bourdillon, A.J., Kabler, M.N., 1976. Intrinsic luminescence excitation spectra in $\mathrm{NaCl}, \mathrm{NaBr}, \mathrm{RbCl}$ and $\mathrm{RbBr}$ between $6 \mathrm{eV}$ and $60 \mathrm{eV}$ using synchrotron radiation. J. Phys. C9, 2961.

Becker, J., Gesland, J.Y., Kirikova, N.Yu., Krupa, J.C., Makhov, V.N., Runne, M., Queffelec, M., Uvarova, T.V., Zimmerer, G., 1998. VUV emission of $\mathrm{Er}^{3+}$ and $\mathrm{Tm}^{3+}$ in fluoride crystals. J. Lumin. 78, 91.

Bel'skii, A.N., Vasil'ev, A.N., Ivanov, S.N., et al., 2016. Optical and luminescent VUV spectroscopy using synchrotron radiation. Crystallogr. Rep. 61, 886.

Chen, Y., Kirm, M., Negodin, E., True, M., Vielhauer, S., Zimmerer, G., 2003. Zero-phonon lines in the d - f luminescence of $\mathrm{LiYF}_{4}: \mathrm{Er}^{3+}$. Phys. Stat. Sol. (b) 240, R1-R3.

Feldbach, E., Jönsson, L., Kirm, M., Kotlov, A., Lushchik, A, A., Nagirnyi, V., Svensson, G., Åsberg-Dahlborg, M., 2000. Polarized emission of $\mathrm{PbWO}_{4}$ and $\mathrm{CdWO}_{4}$ crystals. J. Lumin. 87-89, 1213.

Grigorjeva, L., Pankratov, V., Millers, D., et al., 2001. Transient absorption and luminescence of $\mathrm{LiNbO}_{3}$ and $\mathrm{KNbO}_{3}$. Integ. Ferroelectrics 35, 137.

Gürtler, P., Roick, E., Zimmerer, G., Pouey, M., 1983. SUPERLUMI: a high flux VUV 
spectroscopic device for luminescence measurement. Nucl. Inst. Meth. Phys. Res. 208, 835.

Kan'no, K., 1996. Time-resolved luminescence study on relaxation dynamics and morphology of self-trapped excitons in ionic crystals. J. Elect. Spectr. Rel. Phen. 79, 33.

Khaidukov, N.M., Kirikova, N.Yu., Kirm, M., Krupa, J.C., Makhov, V.N., Negodin, E., Zimmerer, G., 2002. Inter- and intraconfigurational luminescence of $\mathrm{LiYF}_{4}: \mathrm{Er}^{3+}$ under selective VUV excitation. Proc. SPIE 4766, 154.

Kink, R., Löhmus, A., Niedrais, H., Vaino, P., Sorensen, S.L., Huldt, S., Martinson, I., 1991. VUV spectroscopy of solids at low temperature using synchrotron radiation. Phys. Scripta 43, 517.

Kirm, M., Lushchik, A., Lushchik, Ch.B., Vasil'chenko, E, E., 1999a. Relaxation of hot photocarriers created by VUV photons in wide gap crystals. In: The Electrochemical Society Proceedings Series, PV 98-24. pp. 267.

Kirm, M., Zimmerer, G., Feldbach, E., Lushchik, A., Lushchik, Ch.B., Savikhin, F., 1999b. Self-trapping and multiplication of electronic excitations in Al2O3 and Al2O3:Sc crystals. Phys. Rev. B 60, 502.

Kirm, M., Lushchik, A., Lushchik, Ch.B., Zimmerer, G., 2000. Investigation of luminescence properties of pure and $\mathrm{Ce}^{3+}$ doped $\mathrm{Y}_{3} \mathrm{Al}_{5} \mathrm{O}_{12}$ crystals using VUV radiation. In: The Electrochemical Society Proceedings Series, pp. 113 PV 99-40.

Kirm, M., Lushchik, A., Lushchik, Ch.B., Vielhauer, S., Zimmerer, G., 2003a. Luminescence of pure and doped $\mathrm{Al}_{2} \mathrm{O}_{3}$ and $\mathrm{MgO}$ single crystals under inner-shell excitation. J. Lumin. 102-103, 307.

Kirm, M., Vielhauer, S., Zimmerer, G., Kisand, V., Sombrowski, E., Steeg, B., 2003b. Prompt and delayed secondary excitons in rare gas solids. Low Temp. Phys. 29, 822.

Kirm, M., Krupa, J.C., Makhov, V.N., et al., 2004. High-resolution vacuum ultraviolet spectroscopy of 5d - 4f transitions in Gd and Lu fluorides. Phys. Rev. B 70 241101(R).

Kisand, V., Kink, R., Kink, M., Maksimov, J., Kirm, M., Martinson, I., 1996. Low temperature optical spectroscopy of nonlinear BBO crystals. Phys. Scripta 54, 542.

Kotlov, A., 2018. Private Communication, an Excerpt from the Publication Database of the Photon Science at DESY.

Krames, M.R., Shchekin, O.B., Mueller-Mach, R., et al., 2007. Status and future of highpower light-emitting diodes for solid-state lighting. J. Disp. Technol. 3, 160.

Kubota, S., Itoh, M., Ruan, J., et al., 1988. Observation of interatomic radiative transition of valence electrons to outermost-core-hole states in alkali halides. Phys. Rev. Lett. 60, 2319.

Kuzmanoski, A., Pankratov, V., Feldmann, C., 2015. Microwave-assisted ionic-liquidbased synthesis of highly crystalline $\mathrm{CaMoO}_{4}: \mathrm{RE}^{3+}(\mathrm{RE}=\mathrm{Tb}, \mathrm{Sm}, \mathrm{Eu})$ and $\mathrm{Y}_{2} \mathrm{Mo}_{4} \mathrm{O}_{15}: \mathrm{Eu}^{3+}$ nanoparticles. Solid State Sci. 41, 56.

Kuzmanoski, A., Pankratov, V., Feldmann, C., 2016. Energy transfer of the quantumcutter couple $\mathrm{Pr}^{3+}-\mathrm{Mn}^{2+}$ in $\mathrm{CaF}_{2}: \mathrm{Pr}^{3+}, \mathrm{Mn}^{2+}$ nanoparticles. J. Lumin. 179, 555.

Lecoq, P., Korzhik, M., Vasiliev, A., 2014. Can transient phenomena help improving time resolution in scintillators? IEEE Trans. Nucl. Sci. 61, 229.

Lushchik, Ch., Feldbach, E., Frorip, A., et al., 1994. Multiplication of electronic excitations in $\mathrm{CaO}$ and $\mathrm{YAlO}_{3}$ crystals with free and self-trapped excitons. J. Phys. Cond. Matter 6, 11177.

Lushchik, A., Kudrjavtseva, I., Lushchik, Ch., Vasilchenko, E., Kirm, M., Martinson, I., 1995. Creation of stable Frenkel defects by vacuum UV-radiation in KBr crystals under conditions of multiplication of electronic excitations. Phys. Rev. B 52, 1069.

Lushchik, A., Feldbach, E., Kink, R., et al., 1996. Secondary excitons in alkali halide crystals. Phys. Rev. B 53, 5379.

Lushchik, A., Lushchik, Ch., 2018. Evolution of anion and cation excitons in alkali halide crystals. Phys. Sol. State 60, 1487

Makhov, V.N., Kolobanov, V.N., 2001. A conceptual design of the set-up for solid state spectroscopy with free electron laser and insertion device radiation. Nucl. Instrum. Methods Phys. Res. A 467, 1637.

Makhov, V.N., 2014. Vacuum ultraviolet luminescence of wide band-gap solids studied using time-resolved spectroscopy with synchrotron radiation. Phys. Scr. 89, 04401.

Nagirnyi, V., Feldbach, E., Jönsson, L., et al., 1998. Excitonic and recombination processes in $\mathrm{CdWO}_{4}$ and $\mathrm{CaWO}_{4}$ scintillators under synchrotron radiation excitation. Radiat. Meas. 29, 247.

Nagirnyi, V., Feldbach, E., Jönsson, L., Kirm, M., Kotlov, A., Lushchik, A, A., Nagornaya, L.L., Savikhin, F., Svensson, G., 2001. Optical properties of oriented $\mathrm{CdWO}_{4}$ scintillating crystals. Radiat. Meas. 33, 601.

Ogorodnikov, I.N., Omelkov, S.I., Pustovarov, V.A., Kasikov, A., Kirm, M., 2015. Optical and luminescence characterization of $\mathrm{LiBaAlF}_{6}$ single crystals. Opt. Mater. 39, 52.

Olsson, T., Leemann, S.C., Georgiev, G., Paraskaki, G., 2018. Pseudo-single-bunch mode for a $100 \mathrm{MHz}$ storage ring serving soft X-ray timing experiments. Nucl. Instrum. Methods Phys. Res. A 894, 145.

Omelkov, S.I., Nagirnyi, V., Vasil'ev, A.N., Kirm, M., 2016. New features of hot intraband luminescence for fast timing. J. Lumin. 176, 309.

Omelkov, S.I., Nagirnyi, V., Gundacker, S., et al., 2018. Scintillation yield of hot intraband luminescence. J. Lumin. 198, 260.

Pankratov, V., Grigorjeva, L., Chernov, S., et al., 2008. Luminescence properties and energy transfer processes in nanosized cerium doped YAG. IEEE Trans. Nucl. Sci. 55, 1509.

Pankratov, V., Hoszowska, J., Dousse, J.-Cl., et al., 2016. Vacuum ultraviolet excitation luminescence spectroscopy of few-layered $\mathrm{MoS}_{2}$. J. Phys. Cond. Matter 28015301.

Pieterson, van L., Reid, M.F., Wegh, R.T., Soverna, S., Meijerink, A., 2002a. $4 \mathrm{f}^{\mathrm{n}}-4 \mathrm{f}^{\mathrm{n}-1} 5 \mathrm{~d}$ transitions of the light lanthanides: experiment and theory. Phys. Rev. B 65045113.

Pieterson, van L., Reid, M.F., Burdick, G.W., Meijerink, A., 2002b. $4 \mathrm{f}^{\mathrm{n}}-4 \mathrm{f}^{\mathrm{n}-1} 5 \mathrm{~d}$ transitions of the heavy lanthanides: experiment and theory. Phys. Rev. B 65045114.

Pärna, R., Sankari, R., Kukk, E., et al., 2017. FinEstBeaMS - a wide-range FinnishEstonian beamline for materials science at the $1.5 \mathrm{GeV}$ storage ring at the MAX IV laboratory. Nucl. Inst. Meth. Phys. Res. A 859, 83.

Rigler, R., Kristensen, O., Roslund, J., Thyberg, P., Oba, K., Eriksson, M., 1987. Molecular structures and dynamics: beamline for time resolved spectroscopy at the MAX synchrotron in Lund. Phys. Scripta T17, 204.

Romet, I., Buryi, M., Corradi, G., et al., 2017. Recombination luminescence and EPR of Mn doped $\mathrm{Li}_{2} \mathrm{~B}_{4} \mathrm{O}_{7}$ single crystals. Opt. Mater. 70, 184.

Rubloff, G.W., 1972. Far-ultraviolet reflectance spectra and the electronic structure of ionic crystals. Phys. Rev. B 5, 662.

Spassky, D., Omelkov, S., Mägi, H., 2014. Energy transfer in solid solutions $\mathrm{Zn}_{\mathrm{x}} \mathrm{Mg}_{1-\mathrm{x}} \mathrm{WO}_{4}$. Opt. Mater. 36, 1660.

Song, K.S., Williams, R.T., 1996. Self-trapped Excitons. Springer, Berlin.

Shirmane, L., Pankratov, V., 2016. Emerging blue-UV luminescence in cerium doped YAG nanocrystals. Phys. Status Solidi RLL 10, 475.

Shirmane, L., Feldmann, C., Pankratov, V., 2017. Comparing the luminescence processes of $\mathrm{YVO}_{4}: \mathrm{Eu}$ and core-shell $\mathrm{YVO}_{4} @ \mathrm{YF}_{3}$ nanocrystals with bulk- $\mathrm{YVO}_{4}$ :Eu. Physica B 504,80 .

Terekhin, M.A., Vasil'ev, A.N., Kamada, M., Nakamura, E., Kubota, S., 1995. Effect of quenching processes on the decay of fast luminescence from barium fluoride excited by VUV synchrotron radiation. Phys. Rev. B 52, 3117.

Tuomela, A., Pankratov, V., Sarakovskis, A., et al., 2016. Oxygen influence on luminescence properties of rare-earth doped $\mathrm{NaLaF}_{4}$. J. Lumin. 179, 16.

Urpelainen, S., Huttula, M., Balasubramanian, T., et al., 2010. FINEST: a high performance branch-line for VUV photon energy range gas phase studies at MAX-Lab. In: AIP Conference Proceedings 1234. pp. 411.

Vasilev, A.N., Kolobanov, V.N., Kuusman, I.L., Lushchik, C.B., Mikhailin, V.V., 1985. Multiplication of electronic excitations in MgO crystals. Phys. Sol. State 27, 2696.

Vielhauer, S., 2003. Innerschalenanregungen und sekundäre Exzitonen in Edelgasfestkörpern. FB Physik, Univ. Hamburg. DESY report. DESY-THESIS-2003007. HASYLAB.

Wegh, R.T., Donker, H., Oskam, K.D., Meijerink, A., 1999. Visible quantum cutting in $\mathrm{LiGdF}_{4}: \mathrm{Eu}^{3+}$ through downconversion. Science 283, 663.

Yen, W.M., Elias, L.R., Huber, D.L., 1970. Utilization of near- and vacuum-ultraviolet synchrotron radiation for the excitation of visible fluorescences in ruby and $\mathrm{MgO}: \mathrm{Cr}^{3+}$. Phys. Rev. Lett. 24, 1011.

Zimmerer, G., 2006. Luminescence spectroscopy with synchrotron radiation: history, highlights, future. J. Lumin. 119-120, 1.

Zimmerer, G., 1991. Status report on luminescence investigations with synchrotron radiation at HASYLAB. Nucl. Inst. Meth. Phys. Res. A 308, 178.

Zimmerer, G., 2007. SUPERLUMI: a unique setup for luminescence spectroscopy with synchrotron radiation. Radiat. Meas. 42, 859.

\footnotetext{
Institute of Solid State Physics, University of Latvia as the Center of Excellence has received funding from the European Union's Horizon 2020 Framework Programme H2020-WIDESPREAD-01-2016-2017-TeamingPhase2 under grant agreement No. 739508, project CAMART ${ }^{2}$
} 